\title{
Detailed Analysis of Li-rich Giants
}

\author{
J. Gregorio-Hetem, B.V. Castilho and B. Barbuy \\ Universidade de São Paulo, CP 3386, São Paulo, SP, 01060-970, Brazil \\ F. Spite and M. Spite \\ Observatoire de Paris, DASGAL, UMR 8633 CNRS, F-92195 Meudon \\ Cedex, France
}

\begin{abstract}
We have carried out a survey to detect Lithium-rich giants (LRGs). In Castilho et al. (1998) we reported the discovery of five red giants showing a strong $\mathrm{Li}$ I $(\lambda 670.78 \mathrm{~nm})$ line and six other ones showing a moderate Li I line. In this work we present the main results of a detailed analysis of 10 stars from our survey and 6 LRGs selected from the literature.
\end{abstract}

\section{The Program Stars and Observational data}

In our search for Lithium-Rich Giants (LRGs) we prepared a list of candidates with IRAS colours in a precise locus of the [12-25] vs. [25-60] diagram and obtained spectroscopy for more than a hundred of them (Castilho et al. 1998). In this Poster we present the detailed analysis of 10 stars, revealed in this survey to show a strong Li line, as well as 6 other previously known LRGs.

The observations obtained at ESO (Chile) provided high-resolution $(R \sim$ $58000)$ spectra in regions from $535 \mathrm{~nm}$ to $807 \mathrm{~nm}$. The medium-resolution spectra in the range $\lambda \lambda 650-680 \mathrm{~nm}$ were obtained at LNA (Brazil), and OHP (France), with $R \sim 20000$. Photometry in the UBVRI Johnson-Cousins system was obtained at the LNA.

\section{Calculations and Results}

Effective temperatures $T_{\text {eff }}$ were estimated from the photometric calibrations by Bessell et al. (1998) and Lejeune et al. (1998) and checked against excitation equilibrium of $\mathrm{FeI}$ lines. Gravities ( $\log g$ ) were obtained from ionization equilibrium of $\mathrm{Fe} \mathrm{I}$ and $\mathrm{Fe}$ II lines. $[\mathrm{Fe} / \mathrm{H}]$ and microturbulence velocities $\mathrm{v}_{\mathrm{t}}$ were derived from FeI curves-of-growth.

The Li abundances were determined by fitting LTE synthetic spectra to the Li $\lambda 670.78 \mathrm{~nm}$ and $\lambda 610.36 \mathrm{~nm}$ (when available) lines. The non-LTE corrections by Carlsson et al. (1994) were then applied. Model atmospheres employed have been interpolated in the MARCS grids computed by Plez (1992) and Plez (1997, private communication). 
In Castilho et al. (2000) we show that the strength of the Li lines is very dependent on $T_{\text {eff }}$ and very little on gravity. A summary of the results obtained for our sample is reported in Table 1.

Table 1. Stellar parameters and Li abundance for the program stars.

\begin{tabular}{lcccccc}
\hline Object & $T_{\text {eff }}$ & $\log \mathrm{g}$ & {$[\mathrm{Fe} / \mathrm{H}]$} & $\begin{array}{c}v_{t} \\
\mathrm{~km} . \mathrm{s}^{-1}\end{array}$ & $\begin{array}{c}\operatorname{logN}(\mathrm{Li}) \\
\text { ETL }\end{array}$ & NETL \\
\hline HD 4893 & 4057 & 1.8 & 0.2 & 3.0 & 0.45 & 0.78 \\
HD 44889 & 3775 & 0.4 & -0.2 & 1.5 & 0.40 & 0.84 \\
HD 65750 & 3600 & 0.6 & -0.4 & 1.5 & 0.95 & 1.35 \\
HD 90082 & 3686 & 0.0 & -0.2 & 1.2 & -0.10 & 0.18 \\
HD 96195 & 3407 & -0.5 & 0.2 & 1.5 & 0.10 & 0.38 \\
GCSS 577 & 3300 & 0.0 & 0.0 & 2.0 & 0.25 & 0.70 \\
HD 176588 & 3793 & 1.6 & 0.0 & 1.5 & 1.10 & 1.57 \\
iras19012-0747 & 3810 & 1.5 & 0.0 & 1.5 & 2.50 & 2.55 \\
iras19038-0026 & 3600 & 1.0 & 0.0 & 1.0 & 0.30 & 0.56 \\
HD178168 & 4000 & 1.0 & 0.0 & 2.5 & 0.50 & 0.92 \\
HD 787 & 3950 & 1.4 & 0.0 & 1.5 & 2.10 & 2.27 \\
HD 19745 & 4750 & 2.9 & 0.1 & 1.2 & 3.85 & 3.65 \\
HD 30238 & 3925 & 1.4 & 0.0 & 1.5 & 0.80 & 1.20 \\
HD 31993 & 4350 & 2.4 & 0.1 & 3.0 & 1.65 & 1.84 \\
HD 39853 & 3850 & 1.6 & -0.3 & 1.5 & 2.80 & 2.95 \\
HD 95799 & 4900 & 3.2 & 0.0 & 1.5 & 3.20 & 3.05 \\
\hline
\end{tabular}

\section{Conclusions}

LRGs are characterized by IRAS colours indicating the presence of a dust envelope and by a high $\mathrm{Li}$ abundance, but the abundances of other elements are typical of normal red giants. This confirms the hypothesis that LRGs may correspond to an evolutionary stage of normal giants where Li and dust are produced.

\section{References}

Bessell, M. S., Castelli, F., Plez, B. 1998, A\&A 333, 231

Carlsson, M., Rutten, R.J., Brus, J.H.M.J., Shchukina, N.G. 1994, A\&A 288, 860

Castilho, B.V., Gregorio-Hetem, J., Spite, F., Spite, M., Barbuy, B. 1998, A\&AS 127,139

Castilho, B.V., Gregorio-Hetem, J., Barbuy, B., Spite, F., Spite, M. 2000, A\&A, in preparation

Lejeune, T., Cuisinier, F., Buser, R. 1998, A\&A 130, 65

Plez, B., Brett, J.M., Nordlund, A. 1992, A\&A 256, 551 